\title{
A computer program for the analysis of the dynamic bending-torsion coupling in bridges using a mini- computer
}

\author{
R. PICON \\ ETS Ingenieros Industriales, Universidad de Sevilla, Seville, Spain. \\ E. ALARCON \\ ETS Ingenieros Industriales, Universidad Politécnica de Madrid, Madrid 3, Spain
}

The analysis of modes and natural frequencies is of primary interest in the computation of the response of bridges. In this article the transfer matrix method is applied to this problem to provide a computer code to calculate the natural frequencies and modes of bridgelike structures. The Fortran computer code is suitable for running on small computers and results are presented for a railway bridge.

\section{INTRODUCTION}

The analysis of modes and natural frequencies is of primary interest in the computation of the response of bridges. In the case of simple beams in which the load is applied eccentrically the torsion modes are very important (Fig. 1). If the applied loads are through the torsion centre and parallel to one of the principal inertia axes of the section, there is a displacement in the force direction and vibrations can be easily studied.

If the loads have a general direction then the displacement can be obtained adding the components in the two principal directions. In general the load is not at the torsion centre and then, there is a complex phenomenon of bending and torsion (Fig. 2). The method of working is the use of an equivalent system: a force through the torsion centre and a torsion moment in it.

\section{COUPLING THROUGH SHAPE FUNCTIONS}

The energy method is very well adapted to the use of orthogonal shape functions. If $y$ and $\theta$ are the displacement and twist in the torsion centre, the response can be approached setting:

$$
\begin{gathered}
y(x, t)=\sum_{1}^{m} Y_{i}(x) \cdot q_{i}(t) \\
\theta(x, t)=\frac{1}{e_{0}} \sum_{m+1}^{m} Y_{i}(x) \cdot q_{i}(t)
\end{gathered}
$$

This leads to a matrix system as:

$$
\mathbf{A} \ddot{\mathbf{q}}+\mathbf{B q}=0
$$

in which typical elements are:

$$
\begin{aligned}
a_{r i} & =\int_{0}^{L} m Y_{r} Y_{i} \mathrm{~d} x \\
\left(k_{r i}\right)_{f} & =\int_{0}^{L} E I Y_{r}^{\prime \prime} Y_{i}^{\prime \prime} \mathrm{d} x
\end{aligned}
$$

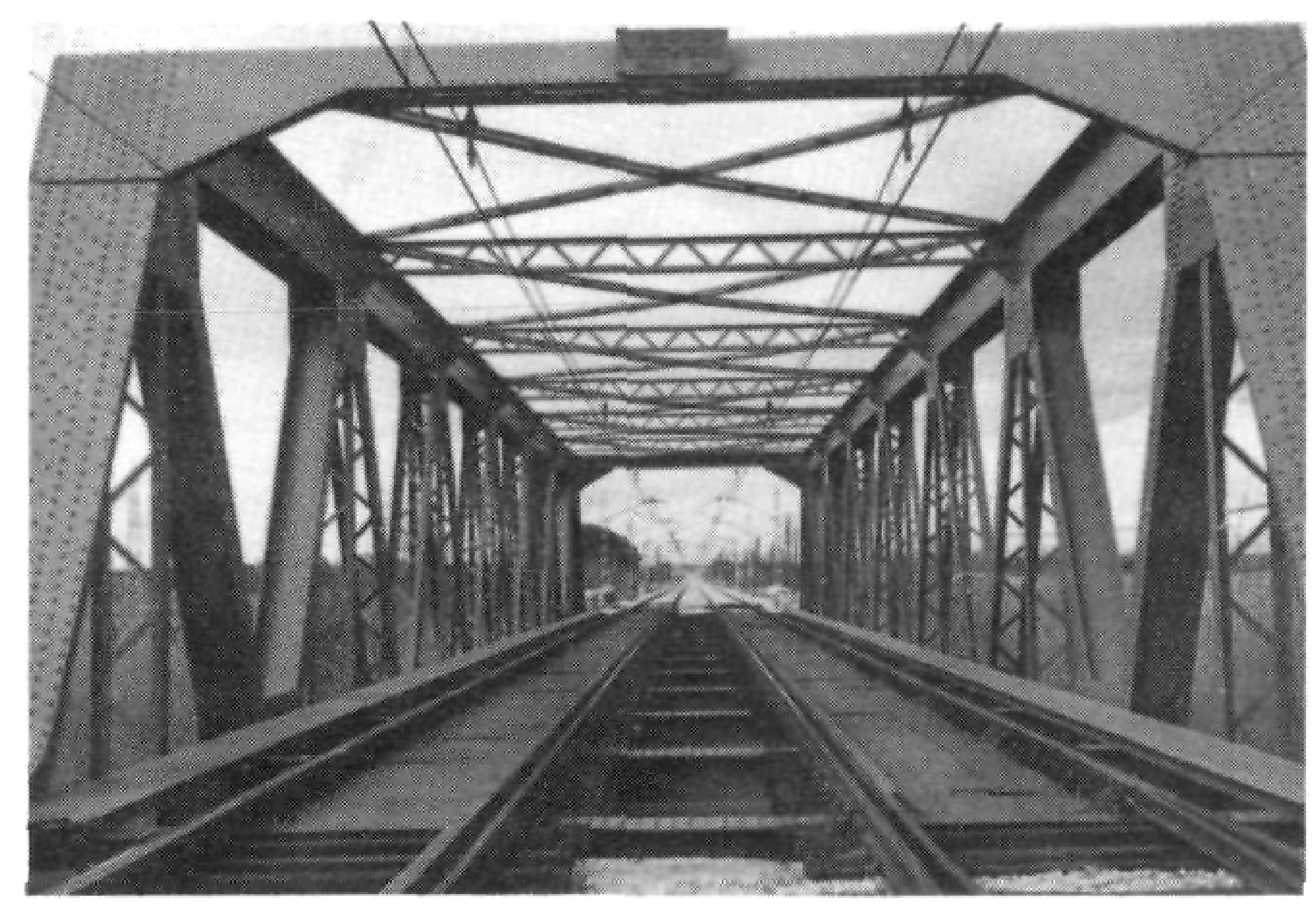

Figure 1.

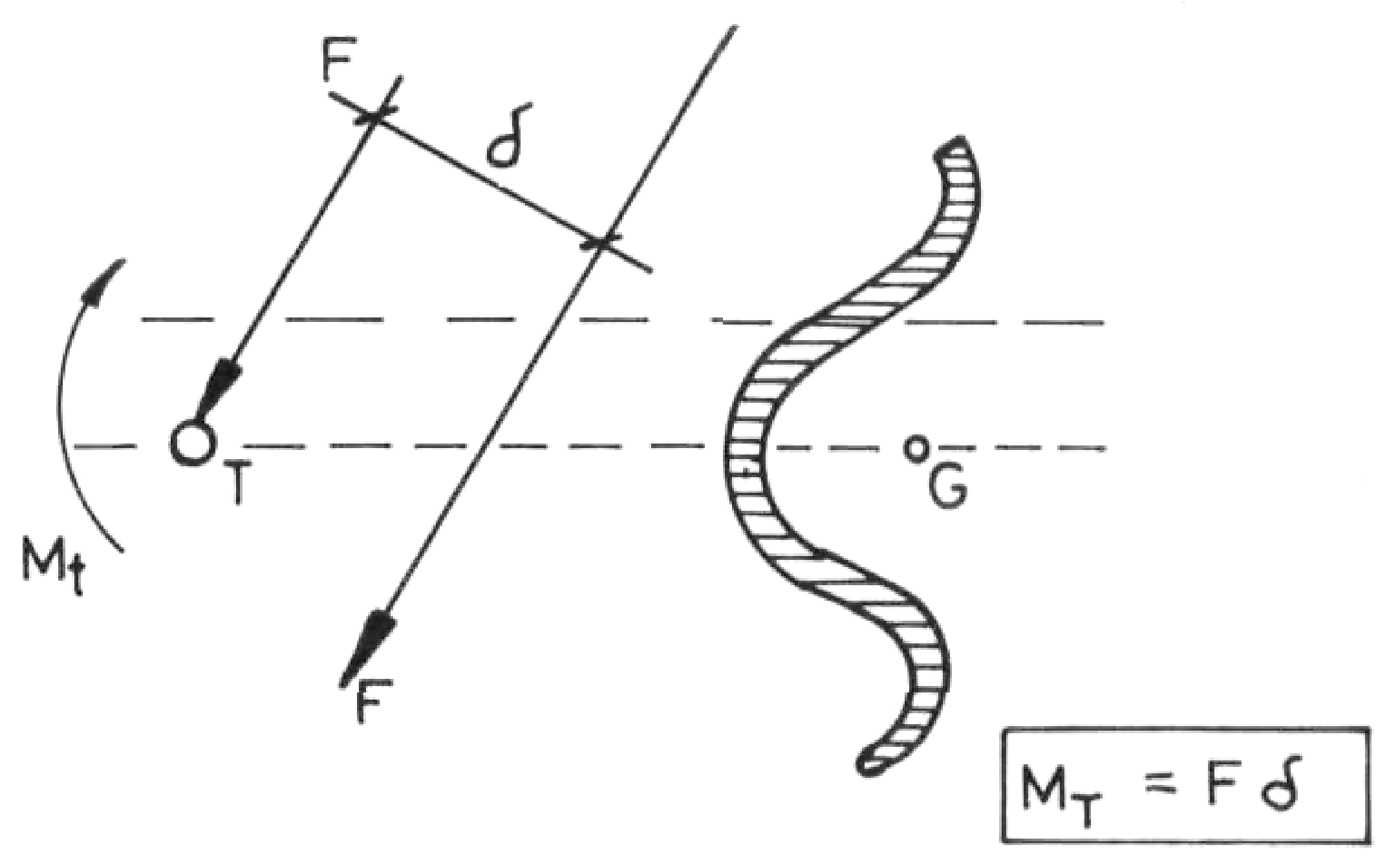

Figure 2. 


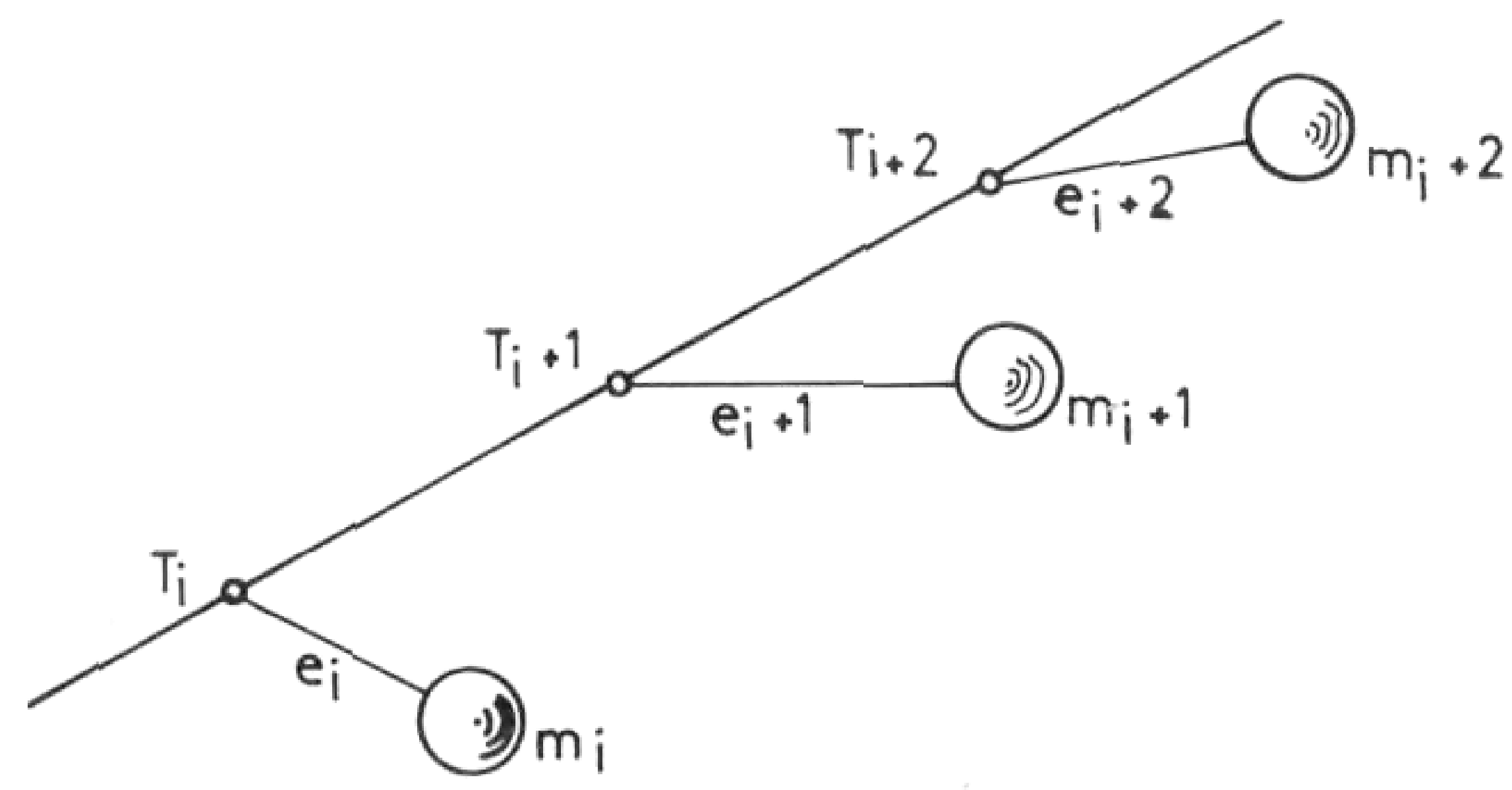

Figure 3.

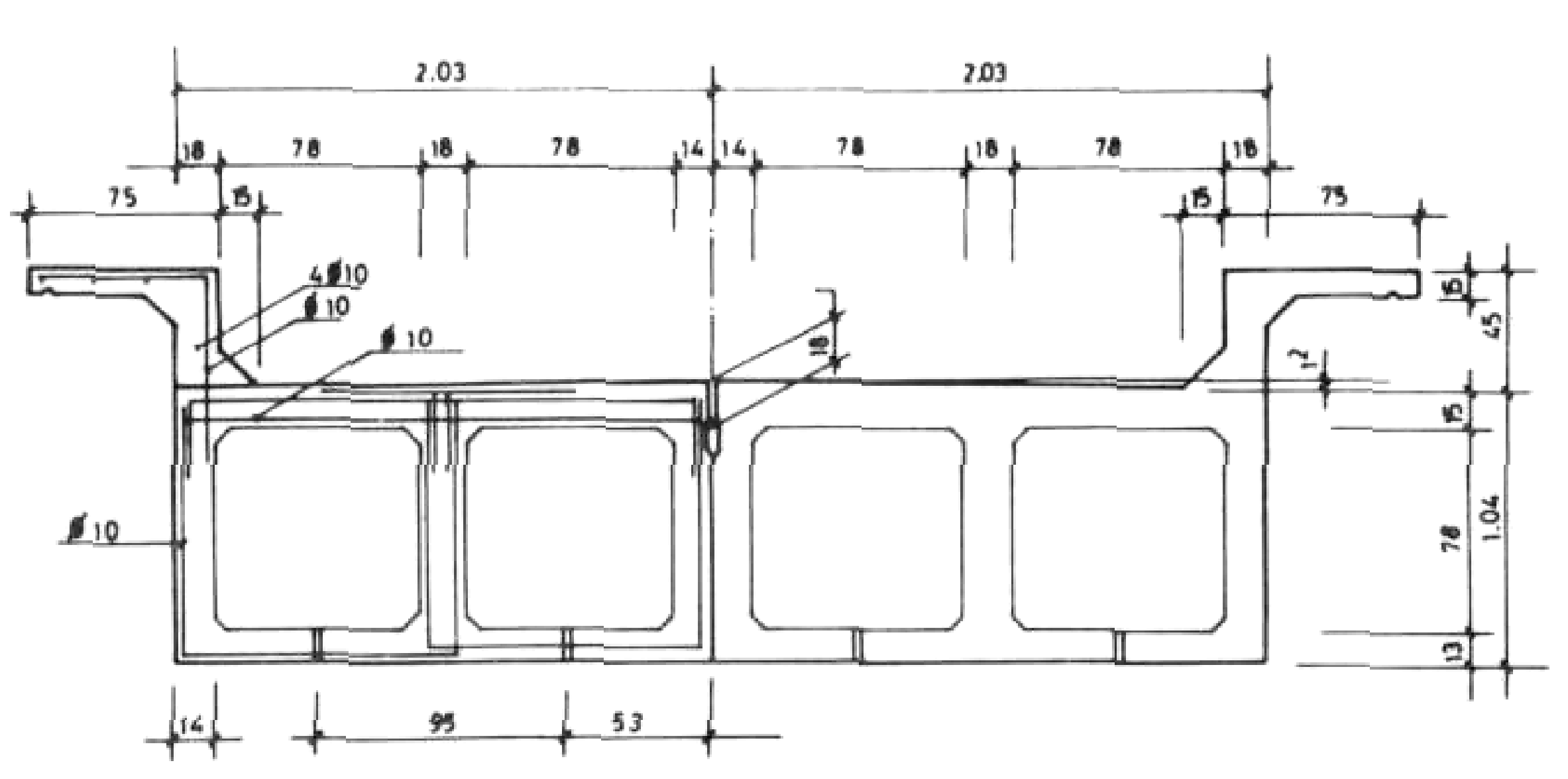

Figure 4.

$$
\left(k_{r i}\right)_{t}=\int_{0}^{L} \frac{G J}{e_{0}^{2}} Y_{r}^{\prime} Y_{i}^{\prime} \mathrm{d} x
$$

The analytical evaluation of integrals is difficult and the derivatives make worse approximation than the original functions.

Nevertheless, as it is well known, the use of locally defined shape functions leads to the finite element method (FEM).

\section{COUPLING THROUGH TRANSFER MATRICES}

The use of transfer matrices ${ }^{1}$ was overpassed by the FEM boom. Nevertheless in some problems, just like this under study, its use can be very fruitful.

As is known, the masses of the beam are lumped in some nodes which are related by elastic bars. In free vibrations loads are only inertial, therefore the general transfer matrix is subdivided in a point matrix which relates forces between both sides of every mass and a field matrix, which refer to both ends of every bar.

If

$$
\mathbf{Z}=\left\{\begin{array}{c}
y \\
\alpha \\
M \\
Q \\
\hdashline-- \\
M_{t}
\end{array}\right\}
$$

contains the characteristic features in every node, then

$$
\mathbf{Z}_{i}^{r}=\mathbf{U}_{p i} \mathbf{Z}_{i}^{l}
$$

where $\mathbf{U}_{p i}$ is the point matrix and the superior index refer to the 'right' and 'left' words.

In every field:

$$
\mathbf{Z}_{i+1}^{l}=\mathbf{U}_{f i} \mathbf{Z}_{i}^{r}=\mathbf{U}_{f i} \mathbf{U}_{p i} \mathbf{Z}_{i}^{l}
$$

If one defines the transfer matrix in section $i$ as:

$$
\mathbf{Z}_{i+1}^{l}=\mathbf{U}_{i} \mathbf{Z}_{i}^{l}
$$

then

$$
\mathbf{U}_{i}==\mathbf{U}_{f i} \mathbf{U}_{p i}
$$

For the simple beam case, the field matrix is:

$$
\mathbf{U}_{i}^{F}=\left(\begin{array}{cccc}
1 & L_{i} & L_{1}^{2} / 2 E I_{i} & L_{i}^{3} / 6 E I_{i} \\
0 & 1 & L_{i} / E I_{i} & L_{i}^{2} / 2 E I_{i} \\
0 & 0 & 1 & L_{i} \\
0 & 0 & 0 & 1
\end{array}\right)
$$

If there is only torsion:

$$
\mathbf{U}_{i}^{T}=\left(\begin{array}{cc}
1 & L_{i} / G J_{i} \\
0 & 1
\end{array}\right)
$$

where $G$ is the shear modulus and $J$ the polar moment of inertia of the bar cross-section.

As the coupling is a dynamic one ${ }^{2}$ it will only appear in the point matrices, so in general:

$U_{c i}=\left(\begin{array}{cccc:cc}1 & L_{i} & L_{i}^{2} / 2 E I_{i} & L_{i}^{3} / 6 E I_{i} & 0 & 0 \\ 0 & 1 & L_{i} / E I_{i} & L_{i}^{2} / 2 E I_{i} & 0 & 0 \\ 0 & 0 & 1 & L_{i} & 0 & 0 \\ 0 & 0 & 0 & 1 & 0 & 0 \\ \hdashline 0 & 0 & 0 & 0 & 1 & L_{i} / G J_{i}^{\prime} \\ 0 & 0 & 0 & 0 & 0 & 0\end{array}\right)$

and

$$
\left\{\begin{array}{c}
y \\
\alpha \\
M \\
Q \\
\theta \\
M_{t}
\end{array}\right\}_{i+1}=U_{c i}\left\{\begin{array}{c}
y \\
\alpha \\
\theta \\
M_{t}
\end{array}\right\}_{i}^{r}
$$

To establish the point matrix it is worth remembering that coupling appears because of the lack of coincidence of the torsion centre and the centroid.

The equilibrium is:

$$
Q_{i}^{r}=Q_{i}^{l}+p^{2} m_{i} y_{i}^{m}
$$




$$
M_{t}^{r}=M_{t}^{l}-p^{2} m_{i} y_{i}^{m} e_{i}-p^{2} J_{i} \theta_{i}
$$

where $y_{i}^{m}=$ centroid displacement, $p=$ natural circular frequency, $e_{i}=$ eccentricity, $J_{i}=$ polar mass moment.

The torsion centre displacement is:

$$
y_{i}=y_{i}^{m}-e_{i}-\theta_{i}
$$

and then

$$
\begin{gathered}
Q_{i}^{r}=Q_{i}^{l}+p^{2} m_{i} y_{i}+p^{2} m_{i} e_{i} \theta_{i} \\
M_{i}^{r}=M_{t}^{l}-p^{2} m_{i} e_{i} y_{i}-p^{2} J_{i} \theta_{i}-p^{2} m_{i} e_{i}^{2} \theta_{i}
\end{gathered}
$$

The point matrix is:

$$
\mathbf{U}_{p i}=\left(\begin{array}{cccc:cc}
1 & 0 & 0 & 0 & 0 & 0 \\
0 & 1 & 0 & 0 & 0 & 0 \\
0 & 0 & 1 & 0 & 0 & 0 \\
p^{2} m_{i} & 0 & 0 & 1 & p^{2} m_{i} e_{i} & 0 \\
\hdashline 0 & 0 & 0 & 0 & 1 & 0 \\
\left(-p^{2} m_{i} e_{i}\right) & 0 & 0 & 0 & -p^{2}\left(m_{i} e_{i}^{2}+J_{i}\right) & 1
\end{array}\right)
$$

The $\mathbf{U}_{c i}$ and $\mathbf{U}_{p i}$ products is the transfer matrix $\mathbf{U}_{i}$ for the $i$ knot (shown in equation 19).
HAMA subroutine, whose argument define the masselement unity.

The only remaining task is to apply the boundary conditions from which the frequency determinant is obtained. The value of the last (RE) is the final product of the program and, when this value is zero, we know that it is a natural mode of vibration.

Though the program is for the analysis of natural modes with coupling, it is also possible to compute bending frequencies (by the residuals 1 to 6 ) or torsion frequencies (by residuals 7 to 9 ).

In these cases it is only necessary to put the eccentricity (EX) equal to zero and to define with a non-zero number (1, for instance) the necessary data to circumvect a divide by zero.

If one is studying non-circular sections it will be necessary to define the adequate connection factor (FACT).

In the circular case FACT $=1$.

As an application we have considered the following boundary conditions $i_{p} e$ : hinged ends for bending and built-in ends for torsion. It is a simple matter to establish other kinds of conditions.

The following step in the program is the change of sign detection in (RE) to get the number of modes established in the input (NFN).

$$
\mathbf{u}_{i}=\left(\begin{array}{cccccc}
\left(1+\frac{p^{2} m_{i} L_{i}^{2}}{6 E I_{i}}\right) & L_{i} & \frac{L_{i}^{2}}{2 E I_{i}} & \frac{L_{i}^{3}}{6 E I_{i}} & p^{2} m_{i} e_{i} L_{i}^{3} & 0 \\
\frac{p^{2} m_{i} L_{i}^{2}}{2 E I_{i}} & 1 & \frac{L_{i}}{E I_{i}} & \frac{L_{i}^{2}}{2 E I_{i}} & p^{2} m_{i} e_{i} L_{i}^{2} & 0 \\
p^{2} m_{i} L_{i} & 0 & 1 & L_{i} & p^{2} m_{i} e_{i} L_{i} & 0 \\
\hdashline p^{2} m_{i} & 0 & 0 & 1 & p^{2} m_{i} e_{i} & 0 \\
\hdashline p^{2} m_{i} e_{i} L_{i} & 0 & 0 & 0 & 1-\frac{p^{2}\left(m_{i} e_{i}^{2}+J_{i}\right) L_{i}}{G} J_{i} & L_{i} \\
\hdashline G J_{i}^{\prime} & 0 & 0 & 0 & -p^{2}\left(m_{i} e_{i}^{2}+J_{i}\right) & 1
\end{array}\right)
$$

It is worth noting that if $e_{i}=0$ there is no coupling and both phenomenon are independent.

\section{COMPUTER PROGRAM}

Using the previous theory a computer program to be used in a Hewlett-Packard 21 MX has been prepared.

There are a variable number of masses, with a maximum of 200 . The program allows $\left(\begin{array}{l}\mathrm{K} 1=1 \\ \mathrm{~K} 1=2\end{array}\right)$ different elements of uniform cross-section. (Numbers in brackets refer to program variables.)

Automatically masses are lumped at the ends of every element. Then mass and constant characteristics of every element are read (D1, D2, D3, D4, D5, D6).

Using the data, transfer matrices are formed, starting with the last mass-element unity (a fictitious element of zero length is added for preventing the symmetry of the procedure). Matrix products gives the transfer matrix which relates the ends of every element.

The transfer matrices formation is completed by the
The INPUT-OUTPUT units are defined by an internal subroutine (RMPAR) which establishes the correspondence between the LOG and INPUT units and between the NPR and the OUTPUT. Variables to define input and output are provided through the keyboard in the same order as they are executed in the program.

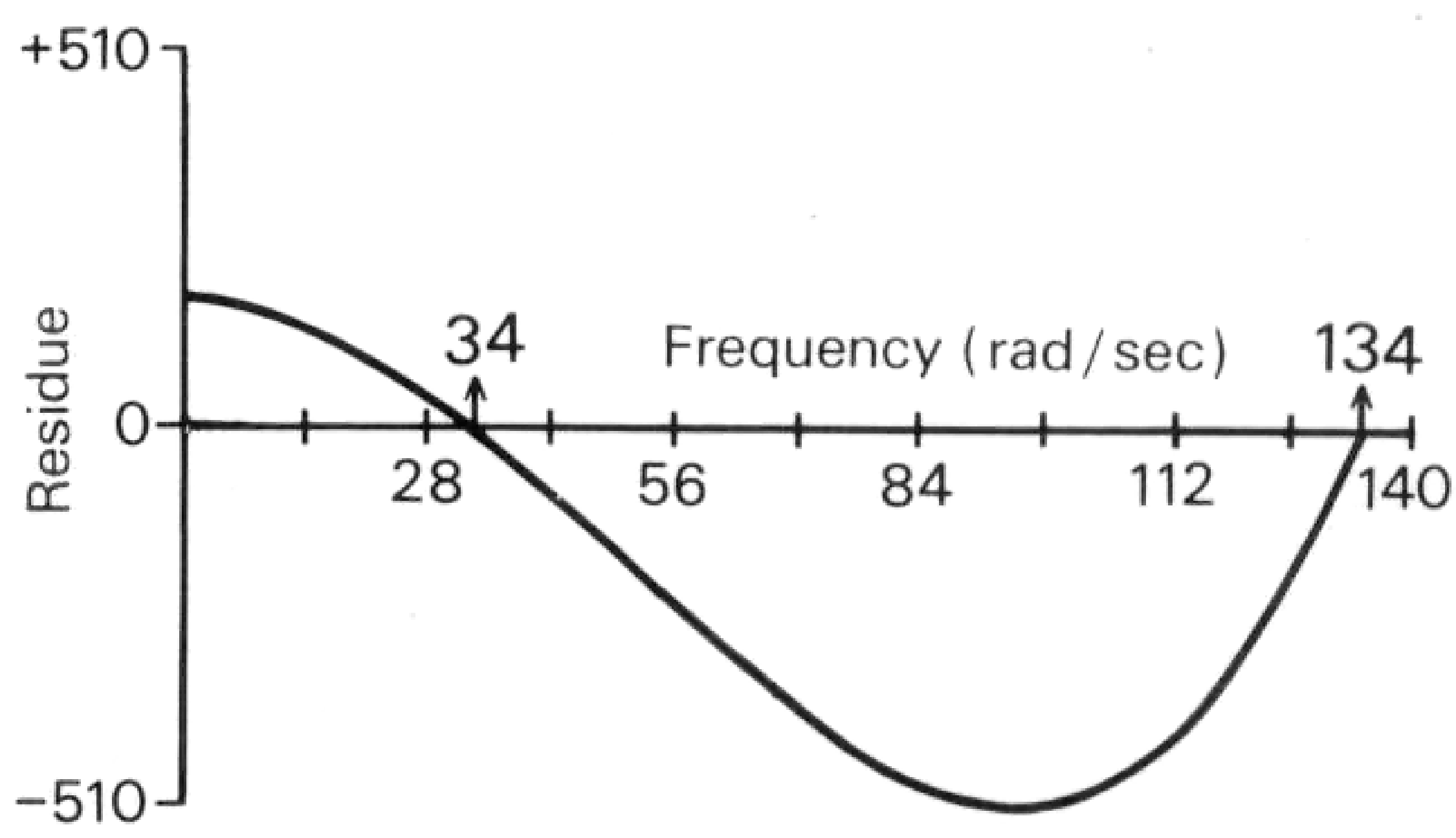

Figure 5. Single track bridge: bending 


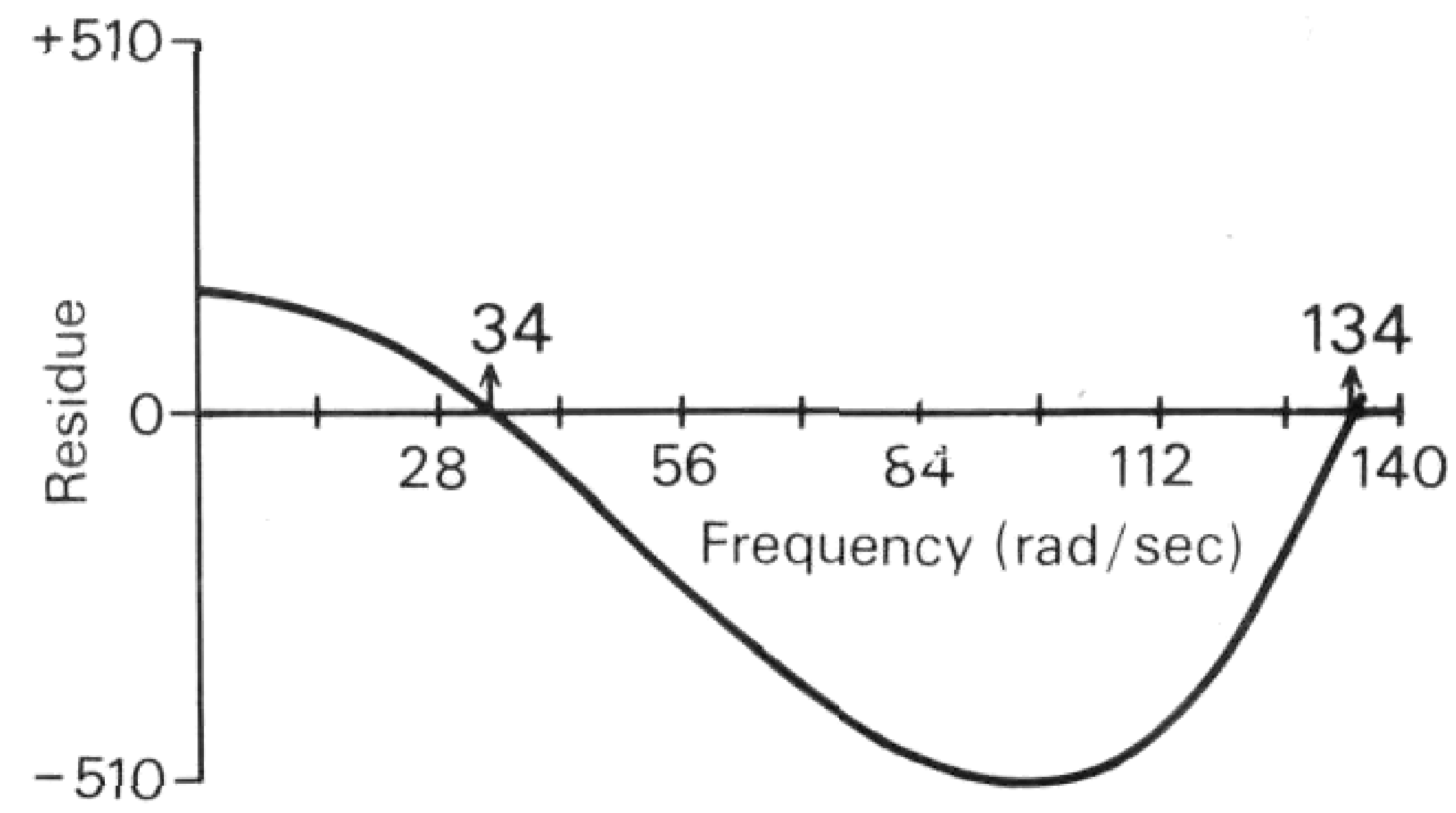

Figure 6. Double track bridge: two trains bending

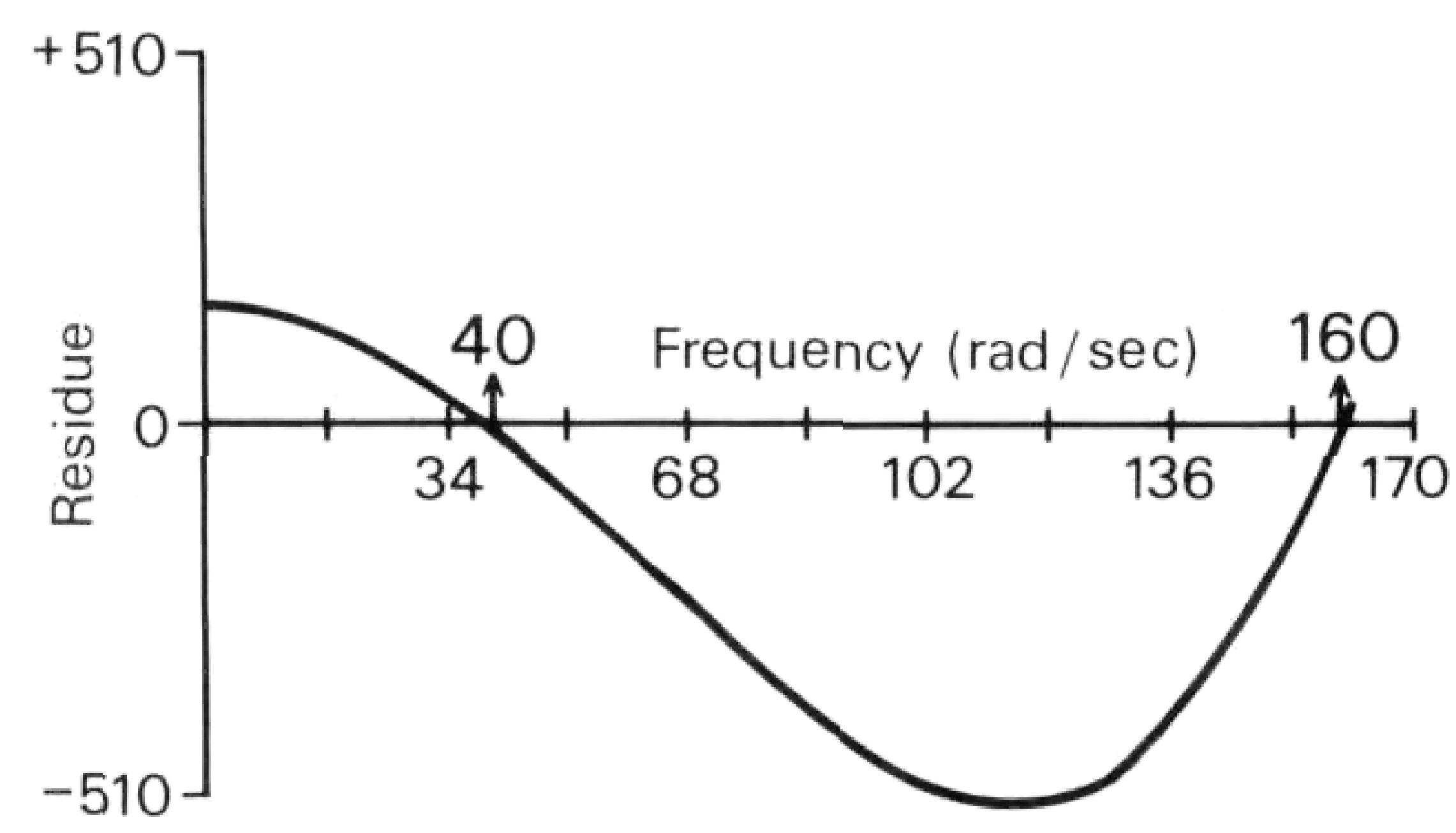

Figure 7. Double track bridge: one train bending $(E X=0)$

\section{Input data}

If $\mathrm{K} 1=1$

1. Header card, columns $1-80$

2. Column 5: K1(1)

Columns 9-10: number of the sought residue, after boundary conditions (see program listing)

3. Columns 8-9-10: number of masses Columns 10-20: number of sought natural modes.

4. Beam length (LT), beam mass (MW), frequency increment (INW), initial frequency (WINI) and correction factor for the inertia polar moment (FACT) in 10 columns fields with point.

5. Inertia bending moment (1), radius of gyration of the masses (RGIR), called D2 in the reading of data), polar moment (MIP). Young's modulus $(E)$, shear modulus $(G)$ and eccentricity (EX) in 10 columns fields with point.

If $\mathrm{K} 1=2$, everything is the same but the 5 th and following cards in which we define the properties of masses and bars in 10 columns fields with point, with a maximum of 7 fields every card.

The input order is: inertia bending moments $(l)$, polar mass moments (MIMPA), polar cross-section moments (MIP), elasticity modulus $(E)$, shear modulus $(G)$, eccentricity $(\mathrm{EX})$, element length $(L)$ and mass $(M)$.

If there are 20 masses there will be 3 cards ( 7,7 and 6) with bending inertias, then 3 polar mass inertias, etc.

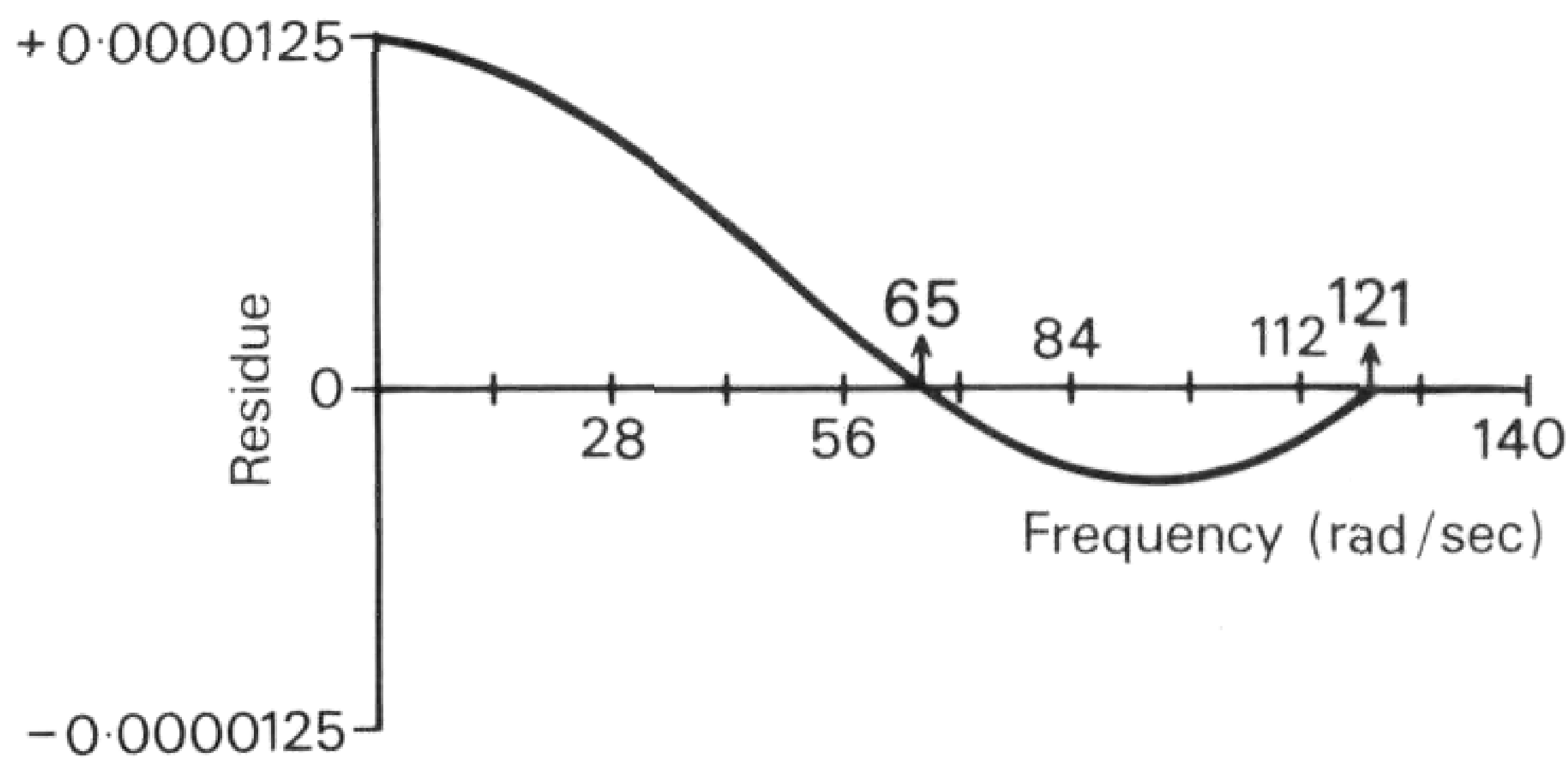

Figure 8. Double track bridge: one train torsion $(E X=0)$

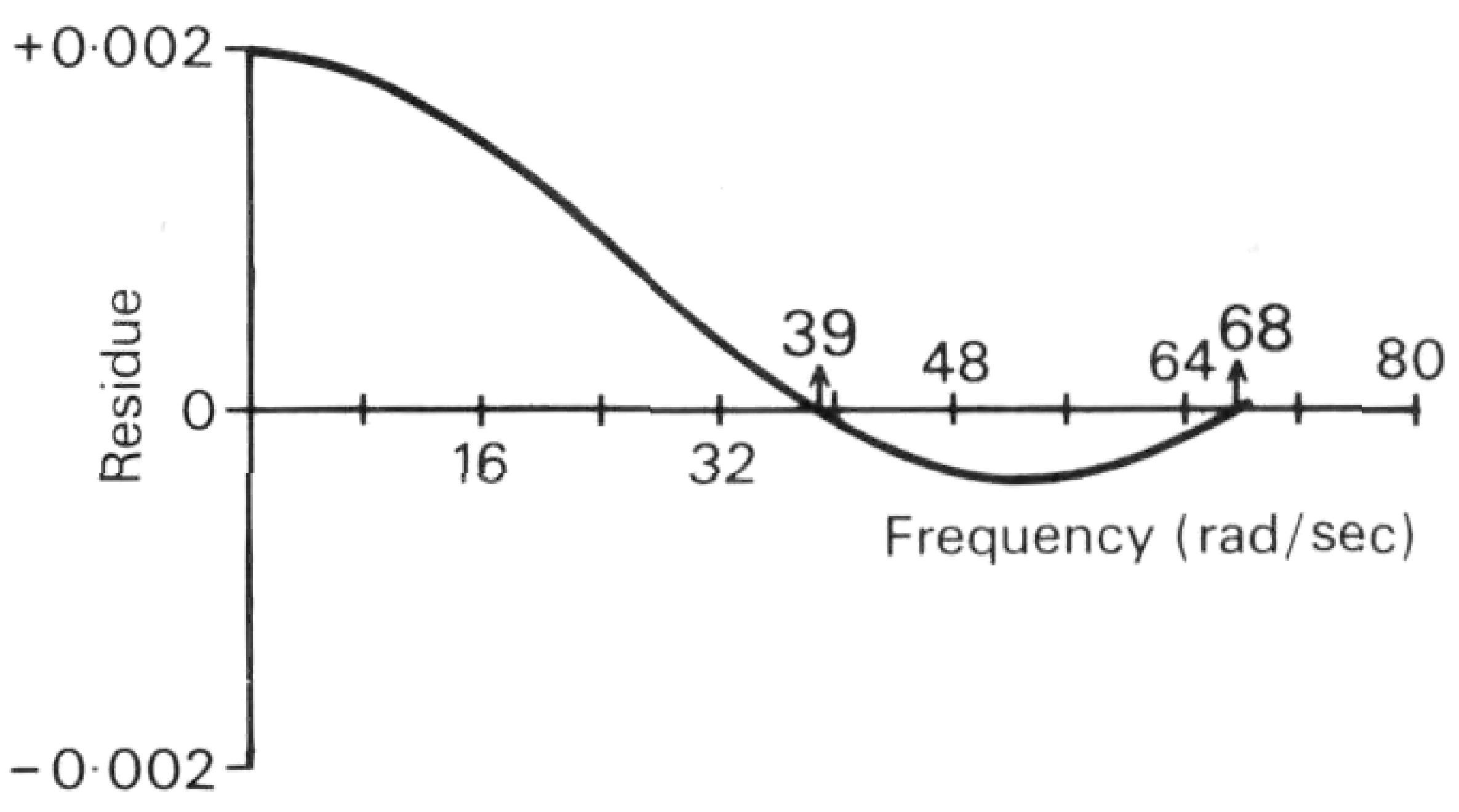

Figure 9. Double track bridge=one bending-torsion coupling $(E X=0)$

\section{Output}

The program produces the following output:

Echo-check of first card and number of masses, listing of frequencies and residuals.

\section{Example}

The example presented here (Fig. 2) corresponds to the characteristics of a prestressed precast bridge of Barton ${ }^{3}$. The Figure is of one-track bridge but a double one will be built with two of them.

The span is $13 \mathrm{~m}$ and we have taken the train-mass as 10 $\mathrm{t} / \mathrm{ml}$. There is also a ballast layer of $40 \mathrm{~cm}$.

The residuals for the first two frequencies have been plotted in Figs. 5, 6, 7, 8, 9. Number of lumped masses was 5 and it is worth noting that an increase to 20 masses affects results very slightly (about 32 ).

\section{REFERENCES}

1 Pestel and Leckie, Matric Methods in Elastomechanics - McGrawHill, New York.

2 Hurty and Rubinstein, Dynamics of Structures, Prentice-Hall, Englewood Cliffs.

3 R. M. Barton, Prestressed precast concrete railroad bridges, $J$. Struct. Div. ASCE, 1968, (12). 
FIMTHI Y=00004 IS DN CROOO18 USING 09029 ELKS R=0256

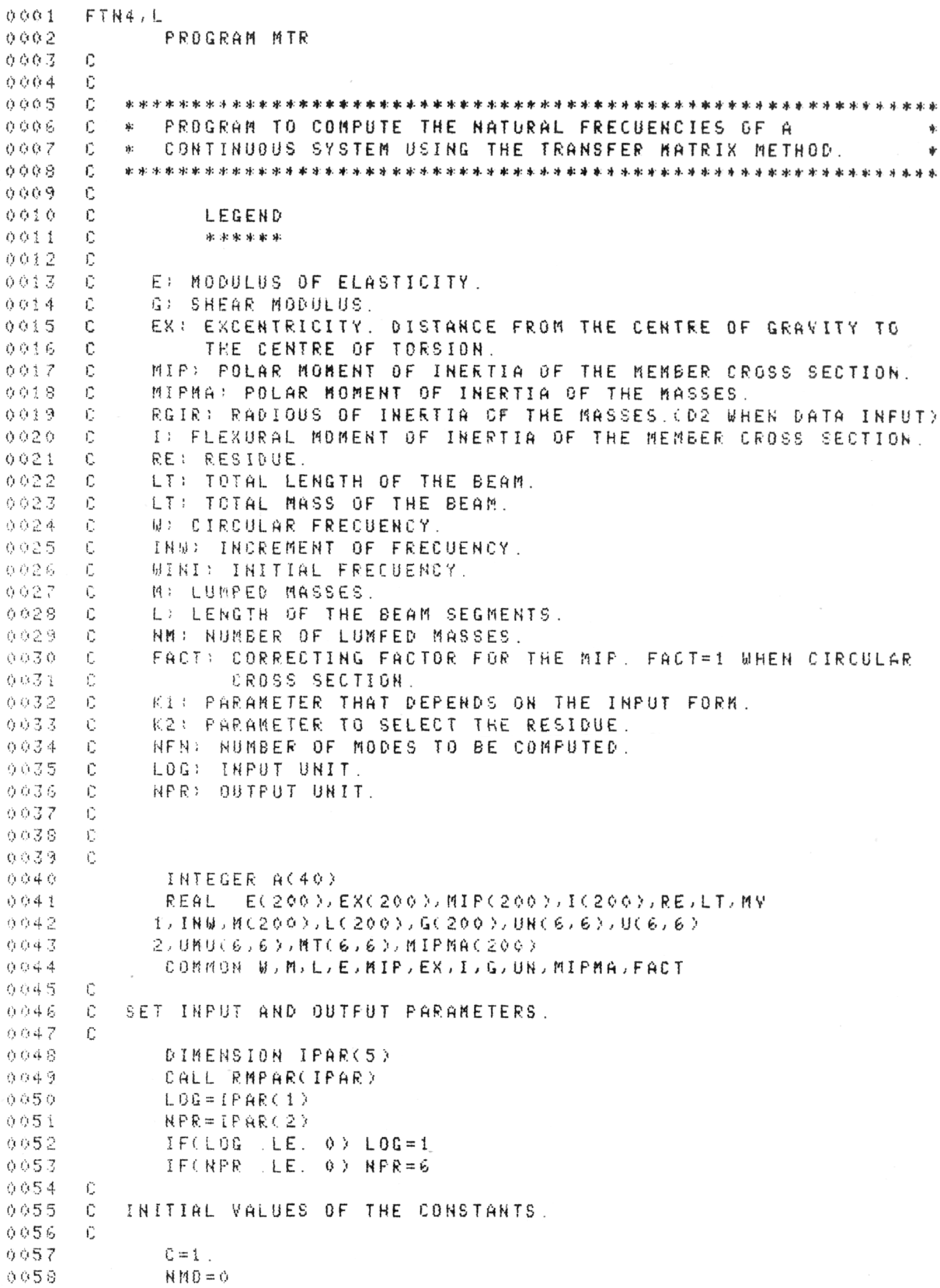




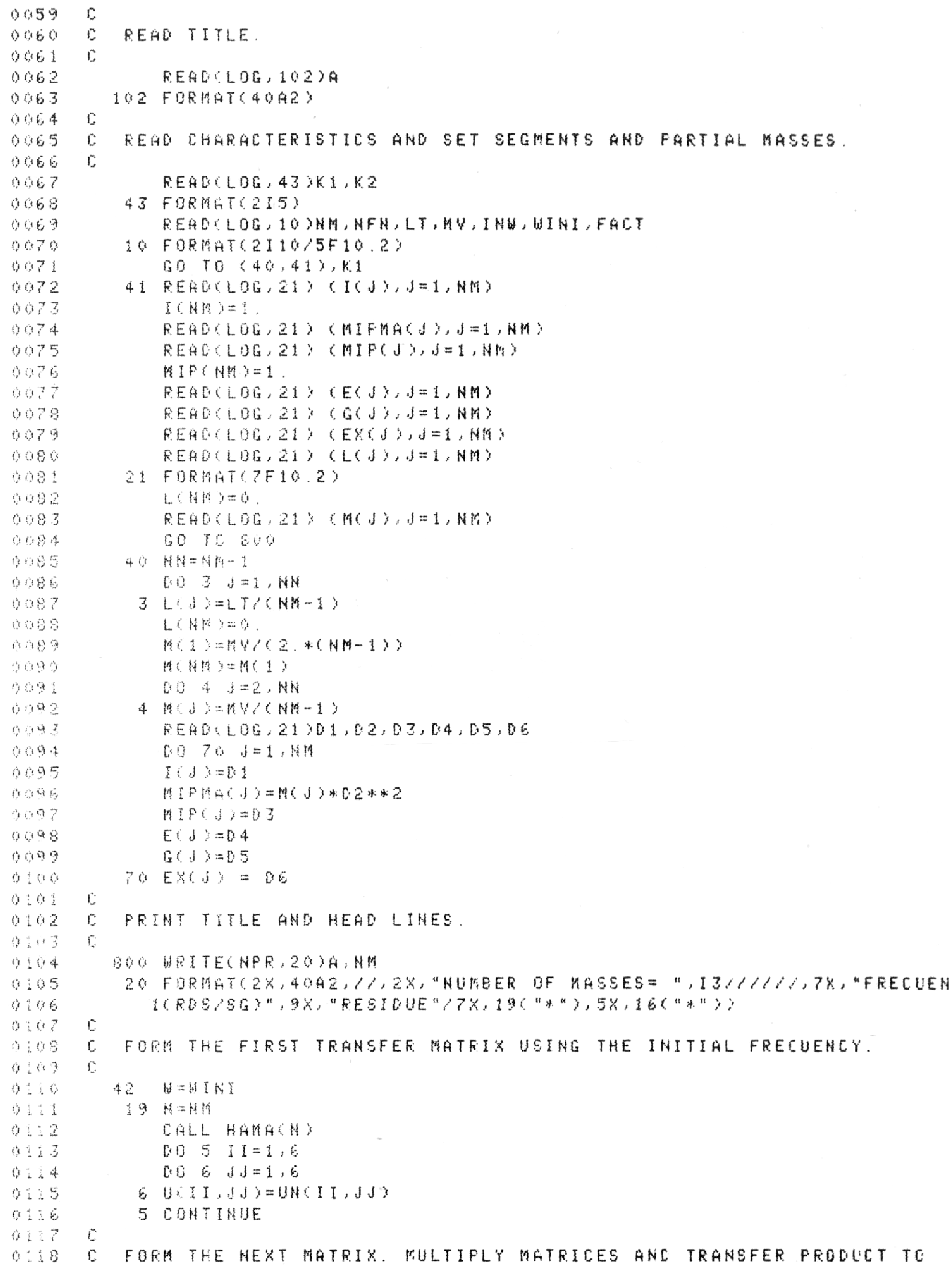




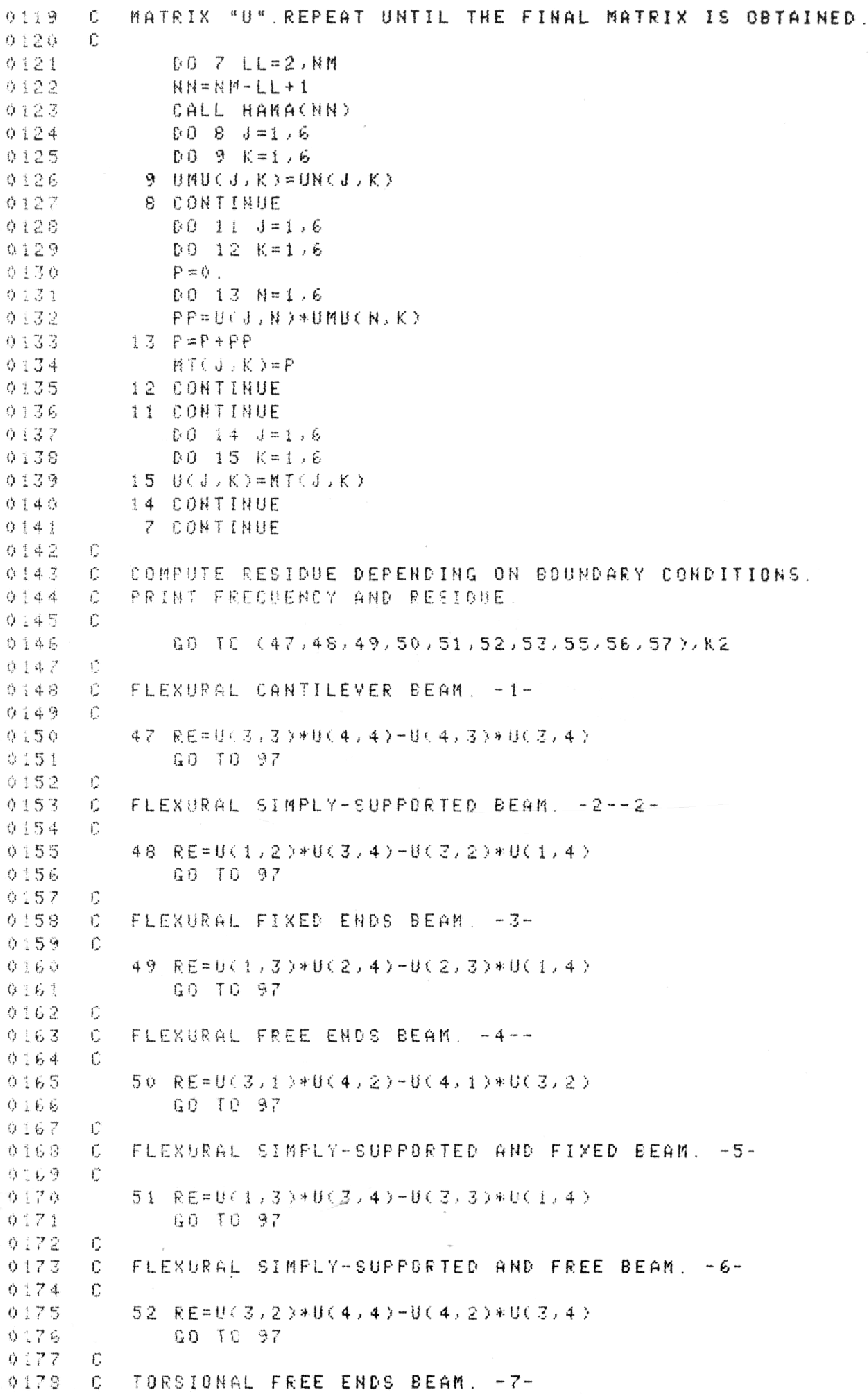




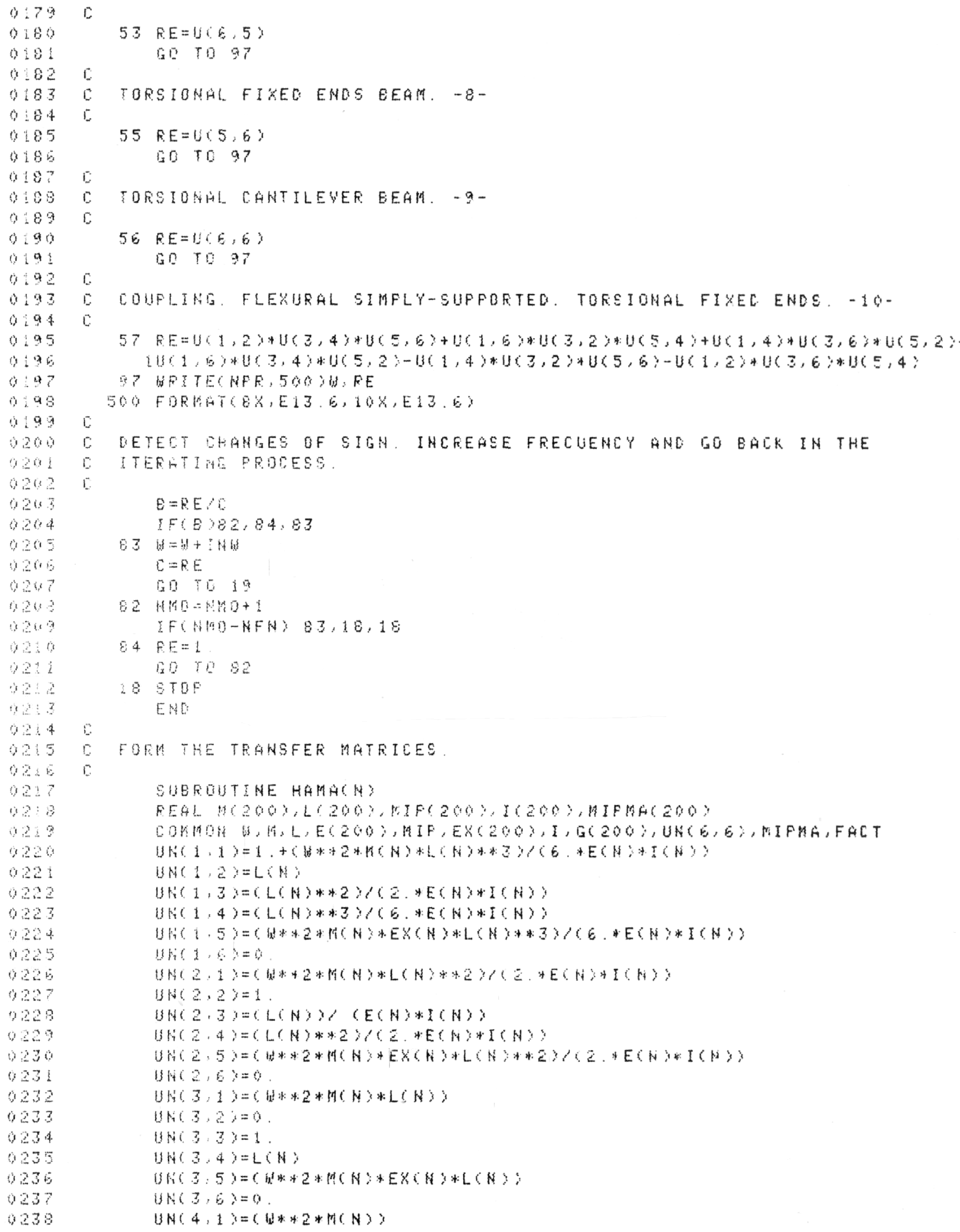




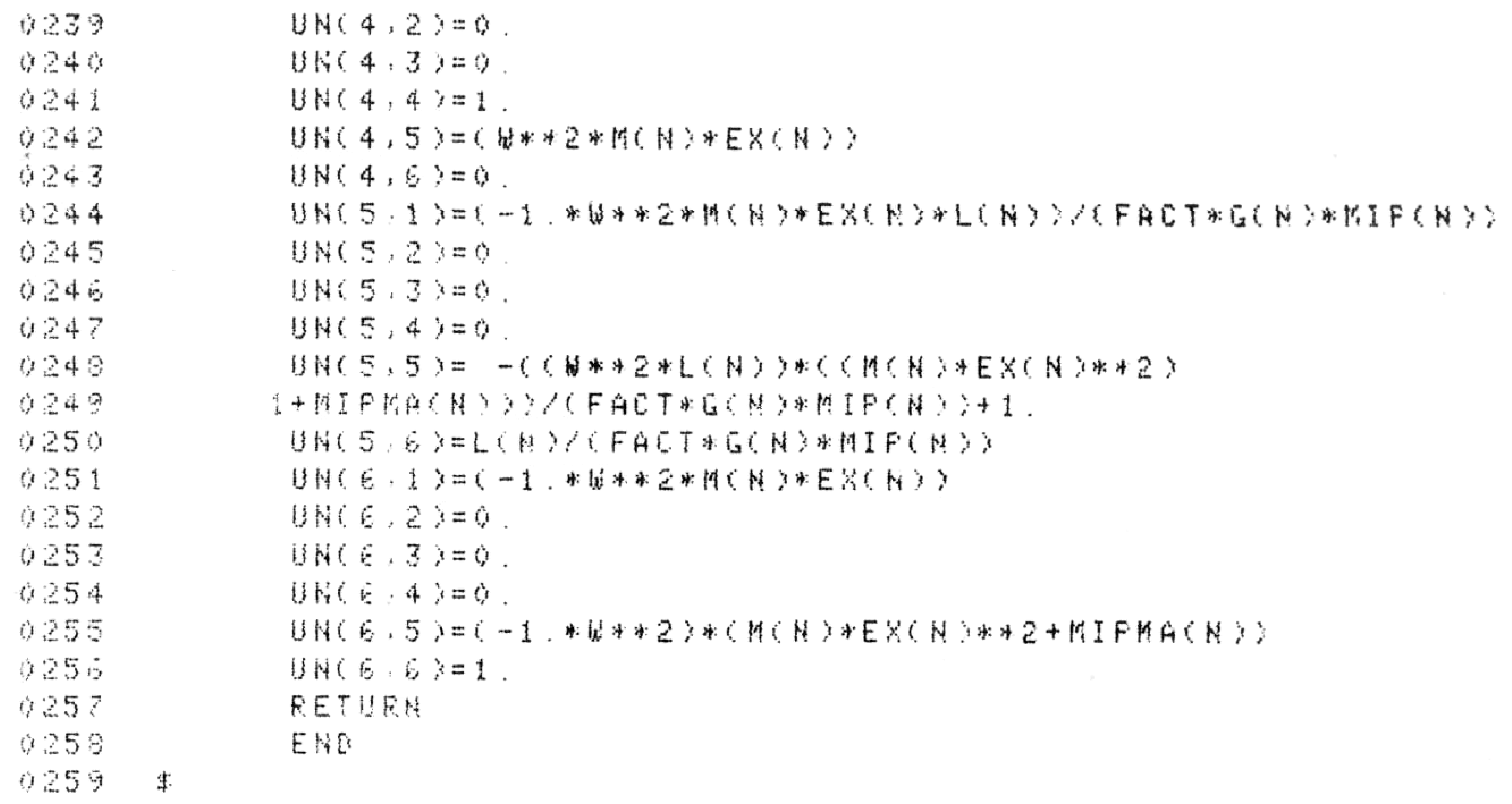

